\title{
Video-Based, Patient-Focused Opioid Education in the Perioperative Period Increases Self-Perceived Opioid-Related Knowledge: A Pilot Study
}

\author{
Johnathan H Goree ${ }^{1}$ \\ Nivetha Srinivasan (D) ${ }^{2}$ \\ Michael A Cucciare ${ }^{3-5}$ \\ Nickolas Zaller ${ }^{6}$ \\ Lauren Byers' \\ Beatrice Boateng ${ }^{7}$ \\ Corey J Hayes ${ }^{4,8}$
}

'Department of Anesthesiology, University of Arkansas for Medical

Sciences, Little Rock, AR, USA; ${ }^{2}$ College of Medicine, Rutgers New Jersey Medical School, Newark, NJ, USA; ${ }^{3}$ Department of Psychiatry, University of Arkansas for Medical Sciences, Little Rock, AR, USA;

${ }^{4}$ Center for Mental Healthcare and

Outcomes Research, Central Arkansas

Veterans Affairs Healthcare System,

North Little Rock, AR, USA; ${ }^{5}$ Veterans

Affairs South Central Mental Illness

Research, Education and Clinical Center,

Central Arkansas Veterans Healthcare

System, North Little Rock, AR, USA; ${ }^{6}$ Fay

W. Boozman College of Public Health,

University of Arkansas for Medical

Sciences, Little Rock, AR, USA

${ }^{7}$ Department of Pediatrics, University of

Arkansas for Medical Sciences, Little

Rock, AR, USA; ${ }^{8}$ Department of

Biomedical Informatics, University of

Arkansas for Medical Sciences, Little

Rock, AR, USA
Correspondence: Johnathan H Goree University of Arkansas for Medical Sciences, Department of Anesthesiology, 430 I West Markham Street, Box 5I5,

Little Rock, AR, 72205, USA

Tel + I 50I-686-88|8

$\mathrm{Fax}+\mid$ 50I-526-72I7

Email Jhgoree@uams.edu
Objective: The aim of this study was to evaluate whether a video-based, patient-focused opioid education tool delivered in the perioperative period would decrease acute and chronic post-operative opioid prescription dispensations and increase self-rated knowledge about opioids.

Methods: We enrolled 110 patients with no reported opioid use in the previous 30 days, undergoing ambulatory surgery for breast, orthopedic, gynecologic, or other outpatient procedures for which opioids would be prescribed. Patients were randomized to receive either post-operative opioid education by the surgical team (control arm) or pre-operative education via a novel 5-minute video plus post-operative education by the surgical team (intervention arm). All patients received follow-up phone calls on post-operative day (POD) 7 to assess self-rated knowledge of opioids on a scale of 1 to 10,10 being most informed, and self-reported opioid use (primary outcome). Arkansas Prescription Drug Monitoring Program (AR PDMP) data were obtained to assess opioid dispensations at POD 90-150 days.

Results: Seventy-seven percent of participants completed POD7 survey. Participants in the intervention arm rated their knowledge of opioids after surgery significantly higher than those in the control arm ( $\mathrm{p}=0.013)$. Data from the AR PDMP reveal trends (non-significant) that show increased use of opioids in the control group when compared to the video intervention group at POD $30(88.7 \%$ vs $76 \%)$ and POD $90-150(22.6 \%$ vs $10 \%)$.

Conclusion: Video-based, patient-focused opioid education can be effectively implemented in a large university hospital during the perioperative period and is effective for increasing a patient's perception of opioid-related knowledge. A fully powered, randomized control trial is needed to further explore observed trends and determine if this novel tool can decrease chronic post-operative opioid dispensations.

Keywords: opioids, perioperative, video education, acute pain, chronic pain, ambulatory surgery

\section{Introduction}

Long-term opioid use often begins with the treatment of acute pain. Many patients develop opioid use disorders after experiencing their first exposure to opioids during a hospital surgical encounter. ${ }^{1}$ Patients are often provided with a prescription for a quantity of opioids not commensurate with their duration of pain without clear instructions on usage, full disclosure of the risk and benefits, or a discussion of alternative treatment strategies for pain control. ${ }^{2,3}$ 
Approximately $6 \%$ of patients who undergo an operative procedure have continued opioid use at 90 days. $^{4}$ While many of these patients have developed chronic postsurgical pain disorders, rates of chronic post-surgical pain are increased by poorly controlled acute post-surgical pain. ${ }^{5,6}$ Recent attempts have been made to standardize perioperative pain control by standardizing post-surgical prescriptions, anesthetic plans, and acute pain regimens. ${ }^{7}$ These attempts have shown a decrease in opioids consumed during the perioperative period, but have not improved patient's post-operative pain scores or patient's perceptions of their pain control. ${ }^{7}$ Thus, interventions are needed to both reduce opioid used in the perioperative period and improve pain control.

Guidelines for opioid prescribing published by the Centers for Disease Control and Prevention (CDC) in 2016 recommend that providers educate patients about the dangers of opioids prior to prescribing them. ${ }^{8}$ Proper opioid education includes risks of long term dependence, potential development of opioid use disorder, harms associated with diversion, dangers of improper disposal, and a discussion of alternative, non-opioid and nonpharmacologic therapies for pain management. Several studies have demonstrated that providing standardized education to patients prior to receipt of an opioid prescription reduces post-operative opioid use and improves surgical outcomes. ${ }^{9-11}$ These studies have primarily used physical handouts or notecards for patient education. There are, however, some limitations with using handouts as an instructional method. Most hospital education materials are produced at a 10th-grade reading level, whereas twenty-two percent of patients read at a 5th-grade reading level or lower. ${ }^{12}$

With known risks of post-surgical acute and chronic opioid use, standardized patient education is needed in the perioperative period to decrease morbidity associated with opioid use and misuse. Since video education has been shown to be superior to handout or verbal education, implementation of video-based opioid education into the perioperative period may be beneficial. ${ }^{13,14}$ By creating a standardized, video-based education tool, which addresses the risks and benefits of acute and chronic opioid use, provides alternative strategies for pain control, and improves overall patient knowledge of safe consumption, storage, and disposal of opioids, one could better disseminate this information during the perioperative period, thereby reducing the risk for long-term chronic pain syndromes and chronic opioid use. ${ }^{4}$ This manuscript describes the development and feasibility of using a novel, video-based, opioid education tool for ambulatory surgery patients in the perioperative period. Our hypothesis is that this novel tool will increase self-rated knowledge of the risks of long-term opioid use and the benefits and alternatives to opioid-based pain management strategies in post-ambulatory surgical patients, as well as decrease chronic post-operative opioid use.

\section{Methods}

\section{Study Design}

A prospective, two-arm, randomized controlled pilot study conforming to the Consolidated Standards of Reporting Trials (CONSORT) guidelines was performed between April 2019 and March 2020 at one academic medical center, The University of Arkansas for Medical Sciences. ${ }^{15}$ The study was approved by the institutional review board (\#228789) and registered at ClinicalTrials.gov (NCT03986866).

\section{Participants}

All ambulatory surgery patients undergoing breast, orthopedic, gynecologic, or other procedures for which opioids would be prescribed were identified via the operative schedule on the day before their procedure by the investigators. The investigators also verified that the patients did not have evidence of opioid prescription in the 30 days prior to surgical intervention via the electronic medical record. Patients were notified of their eligibility by a research team member via phone call on the day prior to the operative procedure. This ensured efficiency during the perioperative period. If interested, on the day of their surgery, patients signed a written consent form and were formally enrolled. All consented patients were undergoing surgical procedures at a university ambulatory surgical center that were not expected to require an overnight admission after the surgical intervention but were presumed to require postoperative opioid therapy by the attending anesthesiologist and surgeon. Participants were not eligible if they were: 1) receiving chronic opioid therapy per our electronic medical record, including opioids to treat opioid use disorder 2) had taken opioids (per patient report) in the previous 30 days, 3) non-English speaking, 4) legally deaf or blind, 5) scheduled for post-operative hospital admission, 6) unable to operate a telephone, and 7) not residents of Arkansas. 


\section{Video Development}

A five-minute educational video was developed with the assistance of a production company based on information from the CDC's "Opioids: What you Need to Know." 16 Prior to completion, the video was reviewed by a multidisciplinary panel of three institutional experts (anesthesiology, psychiatry, and pharmacy) to ensure accuracy (Supplementary Video S1).

\section{Intervention}

After informed consent, patients admitted for ambulatory surgery $(\mathrm{N}=110)$ were randomized to receive either standard of care, opioid education via operative surgeon (control arm; $\mathrm{N}=55$ ), or standard of care plus display of our novel video intervention in the pre-operative holding area (intervention arm; $\mathrm{N}=55$ ). In order to mimic the current perioperative environment, surgeons were advised to provide their routine post-operative education, which is variable depending on surgeon and procedure. Randomization was achieved by drawing pre-shuffled opaque envelopes containing the randomized treatment allocation typed on a piece of paper inside. Patients randomized to the intervention arm were shown a fiveminute video in the pre-operative holding area via a handheld tablet by a member of the research team. Patients randomized to intervention were encouraged to invite their family and/or caretakers to watch the video along with them. Surgeons were not notified of the patient assignment as the research interaction occurred prior to notification from the surgical team that the patient was ready for consent. Surgeons provided opioid analgesia and education to both groups based on their clinical judgement and routine practice.

\section{Post Intervention Follow-Up}

Participants were contacted on post-operative day 7, 30, and 90 , respectively, and were asked a series of questions to obtain their subjective opinion about their chronic pain knowledge, pain control, and the timing of their pain education (Figure 1). For example, on post-operative day 7, patients were asked to rate their knowledge of opioids on a scale from 1 to 10 with 1 being not at all informed and 10 being very informed (primary outcome). Patients were also asked about their self-reported opioid use.

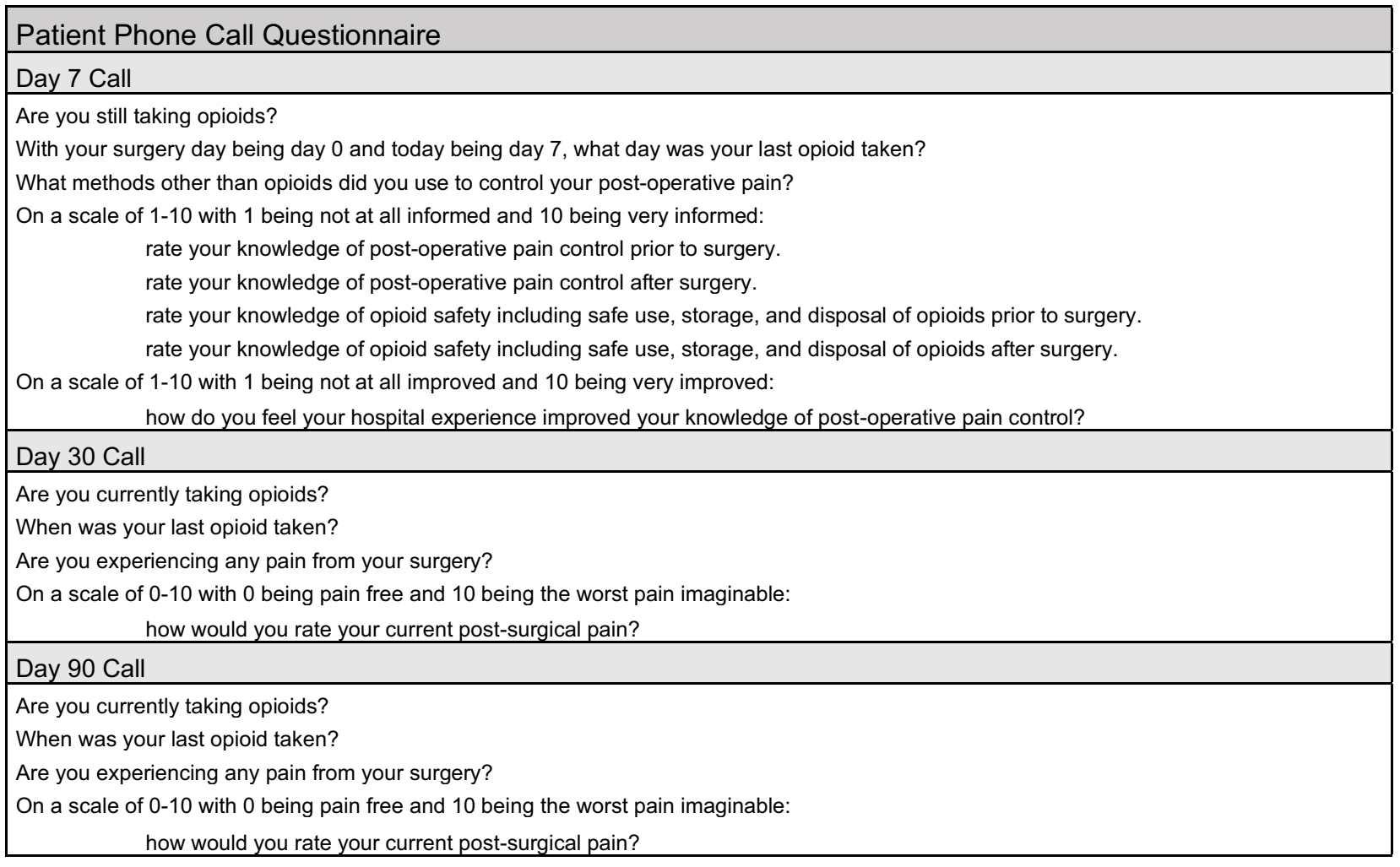

Figure I Questions asked to study subjects on post-operative phone follow-up. 
To assess the effect the intervention had on chronic opioid use, opioid dispensation was used as a surrogate. This was obtained by reviewing AR PDMP data. Since 2013, the AR PDMP, operated by the Arkansas Department of Health $(\mathrm{ADH})$, has captured over 30 million prescription records dispensed for Arkansas residents from pharmacies across Arkansas. AR PDMP provides a nearly comprehensive picture of opioid dispensations among Arkansas residents. Unlike prescribing data, AR PDMP data contain information on what the patient actually received from the pharmacy. AR PDMP data was extracted for study participants using the patient's first name, last name, date of birth, and gender. Opioid dispensations were assessed in the 150 days after the surgery date to ensure adequate time of follow-up and to evaluate long-term opioid therapy. The following specific opioid dispensation metrics were obtained from AR PDMP data: 1) having received any opioid prescription in the 30 days after consent, 2) having received any opioid prescription in the 90-150 days after consent to evaluate persistent opioid utilization (our secondary outcome).

\section{Statistical Analysis}

To evaluate the subjective patient outcomes, the mean, median, and standard deviation for each group were calculated for each question of the survey. Two-tailed t-tests were conducted to determine differences between groups. Patients who were not able to be contacted after four attempts were labeled as lost to follow up. To evaluate the opioid dispensation outcomes by intervention group, logistic regression and generalized linear models were used. Two separate logistic regression models were fit evaluating whether or not the patient received an opioid prescription in the 30 and 90-150 days after surgery. A generalized linear model with a negative binomial distribution was fitted, based on the scale of the variance as compared to the mean, to evaluate the number of opioid prescriptions in the 150 days after the surgery.

\section{Results}

A total of 110 participants were consented and randomized for the study (Figure 2). Of these, four were later excluded because of unforeseen surgical/anesthesia complications requiring surgery cancellation or a post-operative inpatient stay. Three patients formally withdrew from the study after consent and were not included in the analysis. Therefore, 50 patients in the video intervention group and 53 patients in the control arm were included in our analysis. Participants were diverse in age $(45.5 \pm 14.8$ years $)$ and gender $(61 \%$ F). No statistically significant differences (Table 1) in demographics were observed between groups.

\section{Feasibility}

Fifty-five patients were randomized to the intervention arm. Of these patients, all 55 patients (including the 5 patients excluded from statistical analysis) were able to view the video during the perioperative period. Also, none of these 55 patients experienced a surgical delay due to the video intervention as all the intervention was completed prior to surgical consent.

\section{Primary Outcome - Post Intervention Follow-Up}

On post-operative day 7, participants were asked to rate their knowledge of post-operative pain control and to rate their chronic pain knowledge pre- and post-surgery on a scale of $1-10$, with 1 being no knowledge and 10 being complete knowledge (Table 2). The knowledge questionnaire administered during the day 7 post-surgical call showed knowledge of opioid after surgery on a scale of $1-10$ to be $9.364 \pm 1.183$ in the intervention group and $8.319 \pm 2.529$ in the control group ( $\mathrm{p}<0.05$ ). Differences in knowledge of opioids before and after surgery were $1.182 \pm 2.060$ in the intervention group and $0.553 \pm 1.230$ in the control group $(p=0.092)$.

Participants were contacted on post-operative day 7, 30, and 90 and were asked about the last time they took an opioid (Table 3). There were no statistically significant differences in self-reported use of opioids between groups on post-operative day 7, 30, and day 90 .

Pain intensity ratings on post-operative day 30 were also collected for patients in the interventional group (2.11 $\pm 2.98, \mathrm{n}=43)$ and control group $(2.28 \pm 2.64, \mathrm{n}=36)$ as well as on follow-up day 90 for patients in the interventional group $(1.36 \pm 2.53, \mathrm{n}=35)$ and control group (1.73 $\pm 2.41, \mathrm{n}=37)$. There were no statistically significant differences in pain intensity between the groups on postoperative day 30 and day 90.

Variations were observed in which post-operative calls patients attended. Comparable amounts of patients had missing data from the intervention and control group on follow-up day 7 (7 vs 6) and follow-up day 90 (17 vs 14).

\section{Secondary Outcome - Arkansas Prescription Drug Monitoring Database}

Using the AR PDMP, we assessed receipts of postoperative opioid prescription participants from Arkansas 


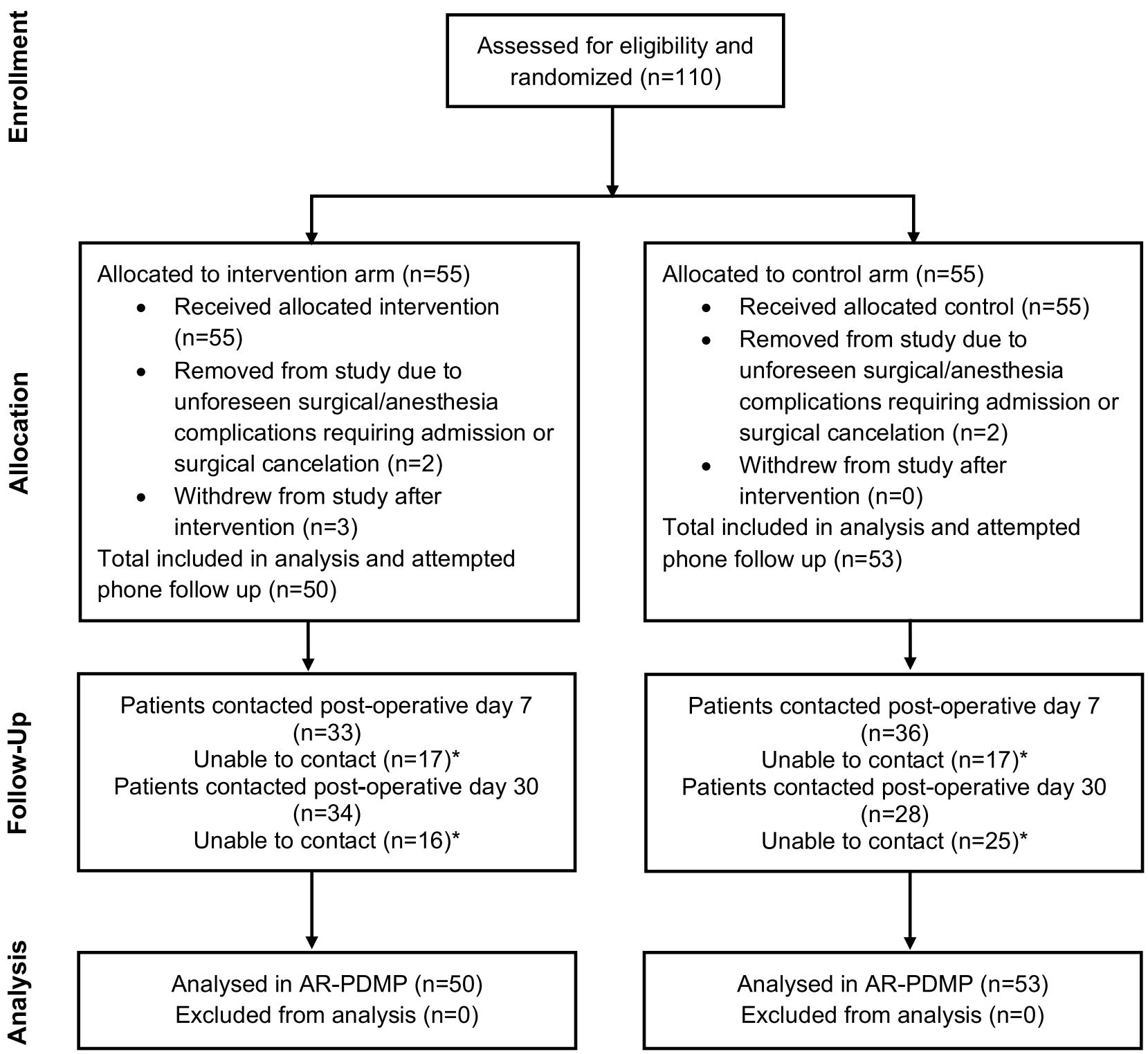

Figure 2 Subject disposition diagram. *Patient labeled unable to contact after 4 attempts to contact by phone. All currently enrolled patients were attempted to contact at each time point.

pharmacies. AR PDMP data revealed $47(89 \%)$ patients in the control arm and $38(76 \%)$ patients in the intervention arm were dispensed a prescription for opioids in the first 30 days post-surgery. AR PDMP data revealed 12 (22\%) patients in the control arm and $5(10 \%)$ patients in the intervention arm were dispensed an opioid prescription during post-operative days 90 to 150 . While both of these showed strong trends, neither was statistically significant (Table 4).

The probability of filling an opioid prescription in the 30 days and $90-150$ days after surgery was not statistically different between the two groups (Figures 3; 30 days: $\mathrm{OR}=0.404, \quad 95 \% \quad \mathrm{CI}: \quad 0.139-1.178 ; \quad 90-150 \quad$ days: $\mathrm{OR}=0.380,95 \%$ CI: $0.123-1.170$ ).

\section{Discussion}

This pilot study examined the feasibility of creating and implementing a novel, patient-focused, video-based opioid education tool in the perioperative period. It examined patient-reported knowledge of opioids and found that patients reported increased knowledge after receiving the intervention. Finally, we examined the effects of this 
Table I Demographic Characterization by Video Randomization

\begin{tabular}{|l|l|l|}
\hline & No Video (n=53) & Video (n=50) \\
\hline Age & & \\
$18-30$ & $6(11 \%)$ & $9(18 \%)$ \\
$31-45$ & $18(34 \%)$ & $16(32 \%)$ \\
$46-60$ & $18(34 \%)$ & $18(36 \%)$ \\
$61-75$ & $8(15 \%)$ & $7(14 \%)$ \\
$76-90$ & $3(6 \%)$ & $0(0 \%)$ \\
\hline Gender & & \\
Men & $24(45 \%)$ & $16(32 \%)$ \\
Women & $29(55 \%)$ & $34(68 \%)$ \\
\hline Race & & $30(60 \%)$ \\
White & $43(81 \%)$ & $20(40 \%)$ \\
Non-White & $10(19 \%)$ & \\
\hline Procedure Type & & $29(58 \%)$ \\
Orthopedic & $32(60 \%)$ & $8(16 \%)$ \\
Gynecology & $8(15 \%)$ & $10(20 \%)$ \\
Breast & $2(4 \%)$ & $3(6 \%)$ \\
Other & $11(21 \%)$ &
\end{tabular}

intervention at reducing opioid dispensations to ambulatory post-surgical patients in the first 30 days after surgery (acute opioid therapy) and between post-operative days 90-150 (chronic opioid therapy). We did not appreciate a statistically significant difference but found a trend that indicated decreased use of chronic opioids in the video intervention group.

First, we found that the creation and implementation of this intervention was feasible. Of the 55 patients who we attempted to show the video to (including 5 not included in the statistical analysis), all were able to view the video during the pre-operative period. Despite the large number of tasks that must be accomplished prior to a surgical encounter (surgical consent, interview and examination by the anesthesiologist, pre-surgical labs, nursing preoperative questionnaire), we found that there was adequate time for every patient to view this 5-minute video in our ambulatory surgery pre-operative holding area without delaying surgical intervention. Patients did not report any inconveniences regarding the timing of enrollment or intervention. Our findings suggest that a 5-minute videobased opioid education intervention was feasible for use in a pre-operative holding area of a large university ambulatory surgery center.

We also found that patients were able to process videobased opioid education. Over one third of US adults have limited health literacy, which includes difficulty reading and understanding health care information on handouts and prescriptions. ${ }^{17}$ Literature has suggested that we should not individually screen for low health literacy because these efforts are often ineffective. Instead, we should ensure that all of our communications with patients avoid medical jargon and provide information in a universal form that is understandable and accessible to all patients. ${ }^{17}$ Other examples of pre-procedural, patientcentered interventions that have been used for reasons other than opioid education are interviews, videos and animations. $^{10,18,19}$ Pre-procedural patient-centered interview interventions were shown to decrease postprocedural pain, improve surgical recovery, and increase daily activity after surgery. ${ }^{10}$ Pre-procedural video-based

Table 2 Comparison of Chronic Pain Knowledge Pre- and Post-Surgery on a Scale of $1-10$

\begin{tabular}{|l|l|l|l|l|}
\hline & & No Video & Video & P-value \\
\hline Knowledge of opioids & Prior to surgery & $7.766 \pm 2.720$ & $8.182 \pm 2.385$ & 0.416 \\
& After Surgery* & $8.319 \pm 2.529$ & $9.364 \pm 1.183$ & $0.013^{*}$ \\
\hline Knowledge of post-operative pain control & Prior to surgery & $7.787 \pm 2.475$ & $7.955 \pm 1.999$ & 0.965 \\
& After Surgery & $8.574 \pm 2.134$ & $8.864 \pm 1.948$ & 0.756 \\
\hline
\end{tabular}

Note: *Indicates statistical significance.

Table 3 Last Self-Reported Opioid Consumption

\begin{tabular}{|l|l|l|l|l|l|}
\hline Post-Operative Day & No Video & N & Video & N & P value \\
\hline 7 & $5.028 \pm 2.021$ & 36 & $4.636 \pm 2.329$ & 33 & 0.46 \\
30 & $10.214 \pm 8.153$ & 28 & $9.353 \pm 9.872$ & 34 & 0.708 \\
90 & $13.933 \pm 17.041$ & 30 & $13.897 \pm 20.912$ & 29 & 0.99 \\
\hline
\end{tabular}


Table 4 Number of Patients Who Were Dispensed an Opioid Prescription

\begin{tabular}{|l|c|c|c|c|c|}
\hline & \multicolumn{2}{|c|}{ Randomization Group } & Odds Ratio & \multicolumn{2}{c|}{ Confidence Interval } \\
\hline Post-Operative & No Video (N=53) & Video (N=50) & Odds Ratio & Lower 95\% Cl & Upper 95\% Cl \\
Day & & & & 0.139 & 1.178 \\
$0-30$ & $47(88.68 \%)$ & $38(76 \%)$ & 0.404 & 0.123 & 1.170 \\
$90-150$ & $12(22.64 \%)$ & $5(10 \%)$ & 0.380 & & \\
\hline
\end{tabular}

education has a demonstrable decrease in anxiety among patients due to increased knowledge about the unknown. Similar findings were experienced in a later study in which patients were randomized to either a 6-minute animation or receive a handout. Those who watched the animation had significantly higher knowledge retention when compared to those that did not. ${ }^{19}$ In another comparative study of video-based patient education vs handouts, those who received video-based instruction were more likely to retain knowledge pertaining to prescription medications, preventing home falls, and adherence to pre-surgical instructions. ${ }^{13,14}$ Our video-based education tool will provide an opportunity to ensure that even those patients with inability to read or low health literacy can access vital opioid education that is needed to improve outcomes and safety.

Patients were also able to process video-based opioid education immediately prior to a surgical encounter. Even though many patients received general anesthesia or monitored anesthesia care within 1-2 hours of viewing the video, it has been noted in the literature that midazolam and other anesthetic drugs primarily cause anterograde amnesia, not retrograde amnesia. ${ }^{20}$ One other pre-study concern was that because this is a high anxiety period, patients may not be able to process important information.
On the contrary, our study demonstrates that opioid education during this period resulted in increased self-rated opioid knowledge. In another study, it was shown that increased preoperative knowledge reduces surgery-related anxiety in spinal stenosis patients. ${ }^{21}$ In another study of patients undergoing orthopedic and neurosurgical procedures, high anxiety about post-operative risks and problems was associated with worse than expected pain experiences. $^{22}$

We also found that our intervention was effective in increasing self-reported knowledge about opioids after an operative encounter. The authors were surprised at the high self-rating of opioid knowledge from both groups. Despite that, current opioid use and the results of this study suggest that patients could be overly confident in their ability to use opioids properly and dispose of them safely. In the majority of cases, post-operative pain is the result of a failure in patient education. We must adequately equip our patients via effective education tools on how to best use opioids and non-opioid modalities postoperatively so that they may have better post-operative pain outcomes.

Finally, we found a trend towards decreased opioid dispensations in the intervention group when compared to the control group. Our feasibility study was not

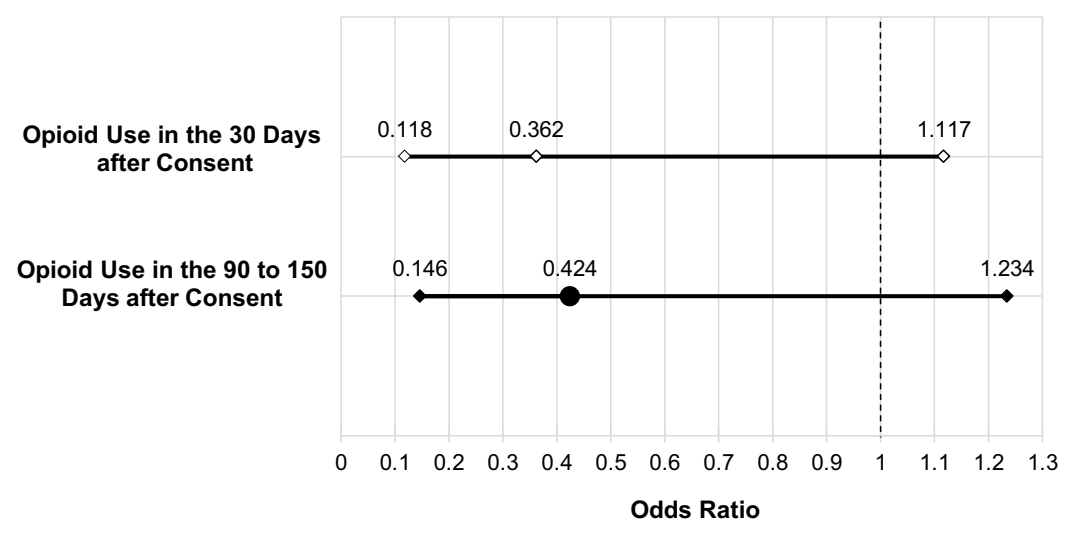

Figure 3 Odds ratio of dispensed prescriptions via Arkansas Prescription Drug Monitoring Database. 
adequately powered to assess this end point. A larger study is needed to further understand whether patient-focused, video-based opioid education in the perioperative period will prevent opioid use after 90 days post-operatively. Since poorly controlled acute pain is correlated with chronic pain states, opioid education tools such as ours may have a larger role in decreasing chronic pain and eventual chronic opioid use. ${ }^{5,6}$

\section{Limitations}

This study had several limitations. First, this was a pilot study and, therefore, not powered to detect differences in our secondary outcomes of outgrowth of opioid therapy and sample size could have eliminated potential bias. Additionally, sample size was further limited by missing follow-up call data for patients. These patients were not able to be considered in the corresponding post-operative day analysis but were included in the PDMP data analysis. Second, while patients were randomized, the surgical team was not notified of patient randomization. Because different surgical teams provided varied levels of perioperative opioid education, this prevented standardization of the control group's level of opioid education. Also, surgeons may have ascertained from patients whether they received the education. While this does present a limitation, it more closely mimics the true in vivo environment, but standardization of prescribing and opioid education in the control group would have been better to evaluate the tool. The authors also only tracked procedure type and not surgeon or post-operative opioid prescriptions. This information would have strengthened the manuscript and will be included in any follow-up studies. The research assistants who performed follow-up calls were not blinded, which could have influenced results. Opioid knowledge was evaluated by self-rating, not a knowledge test. Also, dispensed opioids and day of last consumed opioids were recorded. Measure of number of opioid pills consumed would have been more concise. Surgery type could have influenced the length of opioid treatment. Measure of last consumed opioid medication can be affected by recall. Patients could have had another acute event requiring opioids unknown to the authors that could have affected the data. Finally, our data from the AR PDMP evaluated dispensed opioids. While prescribing behavior is more subject to provider bias, consumed opioids would be most informative.

\section{Conclusion}

This pilot study demonstrates that patient-focused, videobased opioid education can be effectively implemented during the perioperative period in a large, tertiary university hospital ambulatory surgery center. This study also demonstrates that the use of video-based opioid education during the perioperative period is effective at increasing a patient's perception of opioid related knowledge. We also found a trend towards lower opioid dispensations in the intervention group as compared to controls. This pilot study demonstrates the need for a well-powered, randomized, multicenter trial to determine whether this video-based opioid education tool in the perioperative period will decrease postoperative long-term opioid therapy. The authors are constructing a study in the future that will further evaluate this tool against standardized post-operative education and prescribing. In this study, the authors also plan to measure postoperative pill consumption, adherence to prescription directions, and utilizations of alternative pain strategies in addition to the measures previously studied.

\section{Abbreviations}

ARPDMP, Arkansas Prescription Drug Monitoring Program; CONSORT, Consolidated Standards of Reporting Trials; CI, Confidence Interval; OR, Odds Ratio; POD, Post-operative Day.

\section{Data Sharing Statement}

Data are available upon request from the corresponding authors.

\section{Ethics Approval and Informed Consent}

The study was approved by the institutional review board at the University of Arkansas for Medical Sciences $(\# 228789)$ and registered at ClinicalTrials.gov (NCT03986866). The study was conducted in accordance with the Declaration of Helsinki.

\section{Presentation}

The abstract of this paper was presented at the American Society of Regional Anesthesiology and Pain Medicine 2021 Meeting as a poster presentation with interim findings. The paper has not been previously published in a peer-reviewed journal.

\section{Author Contributions}

All authors made a significant contribution to the work reported, whether that is in the conception, study design, execution, acquisition of data, analysis and interpretation, 
or in all these areas; took part in drafting, revising or critically reviewing the article; gave final approval of the version to be published; have agreed on the journal to which the article has been submitted; and agree to be accountable for all aspects of the work.

\section{Funding}

The project described was supported by the University of Arkansas for Medical Sciences Translational Research Institute (TRI), grant U54 TR001629 through the National Center for Advancing Translational Sciences of the National Institutes of Health (NIH). The content is solely the responsibility of the authors and does not necessarily represent the official views of the NIH.

\section{Disclosure}

Dr Johnathan H Goree reports personal fees from Stratus Medical, outside the submitted work, and serves as a consultant for Abbott and Stratus Medical. Miss Nivetha Srinivasan reports grants from the University of Arkansas for Medical Sciences Translational Research Institute, during the conduct of the study. Dr Beatrice Boateng reports grants from NCATS, during the conduct of the study. The authors report no other potential conflicts of interest for this work.

\section{References}

1. Cicero TJ, Ellis MS, Surratt HL, Kurtz SP. The changing face of heroin use in the United States: a retrospective analysis of the past 50 years. JAMA Psychiatry. 2014;71(7):821-826. doi:10.1001/ jamapsychiatry.2014.366

2. Sabatino MJ, Kunkel ST, Ramkumar DB, Keeney BJ, Jevsevar DS. Excess opioid medication and variation in prescribing patterns following common orthopaedic procedures. J Bone Joint Surg Am. 2018;100 (3):180-188. doi:10.2106/JBJS.17.00672

3. Theisen KM, Myrga JM, Hale N, et al. Excessive opioid prescribing after major urologic procedures. Urology. 2019;123:101-107. doi:10.1016/j.urology.2018.06.057

4. Brummett CM, Waljee JF, Goesling J, et al. New persistent opioid use after minor and major surgical procedures in US Adults [published correction appears in JAMA Surg. JAMA Surg. 2017;152(6):e170504. doi:10.1001/jamasurg.2017.0504

5. Gjeilo KH, Stenseth R, Klepstad P. Risk factors and early pharmacological interventions to prevent chronic postsurgical pain following cardiac surgery. Am J Cardiovasc Drugs. 2014;14(5):335-342. doi:10.1007/s40256-014-0083-2
6. Meissner W, Coluzzi F, Fletcher D, et al. Improving the management of post-operative acute pain: priorities for change [published correction appears in Curr Med Res Opin. Curr Med Res Opin. 2015;31 (11):2131-2143. doi:10.1185/03007995.2015.1092122

7. Hartford LB, Van Koughnett JAM, Murphy PB, et al. Standardization of Outpatient Procedure (STOP) Narcotics: a Prospective Non-Inferiority Study to Reduce Opioid Use in Outpatient General Surgical Procedures. J Am Coll Surg. 2019;228(1):81-88.e1. doi:10.1016/j.jamcollsurg.2018.09.008

8. Dowell D, Haegerich TM, Chou R. CDC Guideline for Prescribing Opioids for Chronic Pain-United States, 2016. JAMA. 2016;315 (15):1624-1645. doi:10.1001/jama.2016.1464

9. Hero JO, McMurtry C, Benson J, Blendon R. Discussing Opioid risks with patients to reduce misuse and abuse: evidence from 2 surveys. Ann Fam Med. 2016;14(6):575-577. doi:10.1370/afm.1994

10. Pereira L, Figueiredo-Braga M, Carvalho IP. Preoperative anxiety in ambulatory surgery: the impact of an empathic patient-centered approach on psychological and clinical outcomes. Patient Educ Couns. 2016;99(5):733-738. doi:10.1016/j.pec.2015.11.016

11. Yajnik M, Hill JN, Hunter OO, et al. Patient education and engagement in postoperative pain management decreases opioid use following knee replacement surgery. Patient Educ Couns. 2019;102 (2):383-387. doi:10.1016/j.pec.2018.09.001

12. Safeer RS, Keenan J. Health literacy: the gap between physicians and patients. Am Fam Physician. 2005;72(3):463-468.

13. Shah RF, Gupta RM. Video instruction is more effective than written instruction in improving inhaler technique. Pulm Pharmacol Ther. 2017;46:16-19. doi:10.1016/j.pupt.2017.08.005

14. Ayasrah SM, Ahmad MM. Educational video intervention effects on periprocedural anxiety levels among cardiac catheterization patients: a randomized clinical trial. Res Theory Nurs Pract. 2016;30(1):70-84.

15. Moher D, Hopewell S, Schulz KF, et al. CONSORT 2010 explanation and elaboration: updated guidelines for reporting parallel group randomised trials. Int J Surg. 2012;10(1):28-55.

16. CDC. "Opioids for acute pain: what you need to know". Available from: cdc.gov/drugoverdose/pdf/patients/Opioids-for-Acute-Pain-a. pdf. Accessed April 6, 2020.

17. Hersh L, Salzman B, Snyderman D. Health Literacy in Primary Care Practice. Am Fam Physician. 2015;92(2):118-124.

18. Chakravarthy B, Somasundaram S, Mogi J, et al. Randomized pilot trial measuring knowledge acquisition of opioid education in emergency department patients using a novel media platform. Subst Abus. 2018;39(1):27-31. doi:10.1080/08897077.2017.1375061

19. Hill AM, McPhail S, Hoffmann T, et al. A randomized trial comparing digital video disc with written delivery of falls prevention education for older patients in hospital. J Am Geriatr Soc. 2009;57 (8):1458-1463. doi:10.1111/j.1532-5415.2009.02346.x

20. Bauer KP, Dom PM, Ramirez AM, O'Flaherty JE. Preoperative intravenous midazolam: benefits beyond anxiolysis. J Clin Anesth. 2004;16(3):177-183. doi:10.1016/j.jclinane.2003.07.003

21. Kesänen J, Leino-Kilpi H, Lund T, Montin L, Puukka P, Valkeapää K. Increased preoperative knowledge reduces surgery-related anxiety: a randomised clinical trial in 100 spinal stenosis patients. Eur Spine J. 2017;26(10):2520-2528. doi:10.1007/s00586-017-4963-4

22. Robleda G, Sillero-Sillero A, Puig T, Gich I, Baños JE. Influence of preoperative emotional state on postoperative pain following orthopedic and trauma surgery. Rev Lat Am Enfermagem. 2014;22 (5):785-791. doi:10.1590/0104-1169.0118.2481 


\section{Publish your work in this journal}

The Journal of Pain Research is an international, peer reviewed, open access, online journal that welcomes laboratory and clinical findings in the fields of pain research and the prevention and management of pain. Original research, reviews, symposium reports, hypothesis formation and commentaries are all considered for publication. The manuscript management system is completely online and includes a very quick and fair peer-review system, which is all easy to use. Visit http:// www.dovepress.com/testimonials.php to read real quotes from published authors.

Submit your manuscript here: https://www.dovepress.com/journal-of-pain-research-journal 